\title{
Characterization of disturbance sources for LISA: torsion pendulum results
}

\author{
L Carbone $^{a}$, A Cavalleri ${ }^{b}, \mathbf{R}$ Dolesi ${ }^{a}, \mathbf{C}$ D Hoyle ${ }^{a} \dagger$, \\ M Hueller ${ }^{a}$, S Vitale ${ }^{a}$ and W J Weber ${ }^{a}$ \\ ${ }^{a}$ Dipartimento di Fisica, Università di Trento and INFN, Sezione di Padova, \\ Gruppo Collegato di Trento, I-38050, Povo, Trento, Italy \\ ${ }^{b}$ CEFSA-ITC, I-38050, Povo, Trento, Italy \\ E-mail: carbone@science.unitn.it
}

\begin{abstract}
A torsion pendulum allows ground-based investigation of the purity of free-fall for the LISA test masses inside their capacitive position sensor. This paper presents recent improvements in our torsion pendulum facility that have both increased the pendulum sensitivity and allowed detailed characterization of several important sources of acceleration noise for the LISA test masses. We discuss here an improved upper limit on random force noise originating in the sensor. Additionally, we present new measurement techniques and preliminary results for characterizing the forces caused by the sensor's residual electrostatic fields, dielectric losses, residual spring-like coupling, and temperature gradients.
\end{abstract}

PACS numbers: $04.80 \mathrm{Nn}, 07.10 \mathrm{Pz}, 07.87+\mathrm{v}, 95.55 \mathrm{Yn}$

\section{Introduction}

Achieving the LISA gravitational wave sensitivity requires that the test masses (TM) are kept in free fall with residual accelerations below $3 \mathrm{fm} \mathrm{s}^{-2} \mathrm{~Hz}^{-1 / 2}$, in the low frequency band from $0.1 \mathrm{~Hz}$ down to $0.1 \mathrm{mHz}$ [1]. This is achieved by a Drag-Free control scheme, where external disturbances are shielded by the spacecraft, which is driven to be centered about the freely-falling TM according to a Gravitational Reference Sensor (GRS). In a high feedback loop regime with gain $\omega_{D F}^{2}$, the TM acceleration noise $a_{n}$ can be divided in two main categories, as shown as follows:

$$
a_{n}=\frac{f_{s t r}}{m}+\omega_{p}^{2}\left(x_{n}+\frac{F_{s t r}}{M \omega_{D F}^{2}}\right)
$$

Here, position independent stray forces $f_{\text {str }}$ directly act on the TM (mass $m$ ), whereas spring-like couplings $k_{p}=m \omega_{p}^{2}$ ("stiffness") between the TM and the spacecraft (mass M) couples the residual relative motion, due to position sensor noise $x_{n}$ or external forces $F_{\text {str }}$ acting on the spacecraft, into acceleration noise.

Gravitational Reference Sensors, based on a capacitive readout and actuation scheme, have been developed to meet LISA requirements in terms of both position and acceleration noise [2, 3]. In the ground-testing programme, proceeding in parallel to the design of the LISA flight test LTP [4], a torsion pendulum facility has been developed and is used to validate the functionality of capacitive position sensor

$\dagger$ Presently at University of Washington, Seattle (WA), USA 
prototypes and, most importantly, to investigate the purity of free fall allowed by the GRS [5, 6, 7]. Moreover, with its high force sensitivity this facility is also used to accurately characterize several possible disturbance sources that could prevent the achievement of the LISA goal.

Recent studies [6, 7] have already put significant upper limits on surface stray forces, including those related to the read-out back-action electrostatic noise and molecular impacts. Additionally, the most significant source of spring-like coupling, the $\mathrm{AC}$ voltage bias used for the capacitive position readout, has been measured and found to agree with the theoretical model prediction at the $10 \%$ level. The need for a more detailed characterization of the GRS focuses current experimental activity on improving the torsion pendulum noise and extending its sensitivity to lower frequencies. Moreover, it investigates several noise sources which have been identified as threatening to LISA.

In this paper we report on most recent results of this experimental campaign. In the first section we will focus on the upgrades of the facility. Then we will discuss the most recent upper limits on stray forces, allowed by the pendulum's improved sensitivity. Finally, we will describe measurement techniques developed to investigate stray electrostatics effects in the GRS, like electrical patch fields or dielectric losses, thermal gradients related effects and residual spring-like couplings.

\section{Apparatus Design}

A detailed description of the entire facility is given in [7. The torsion pendulum is comprised of a representative copy of a LISA TM hanging from a long thin fiber inside a Mo-Shapal GRS prototype [2, 3, all inside a high vacuum chamber. We use a hollow cubic TM, made of Au-coated Ti, with $40 \mathrm{~mm}$ side length and $2 \mathrm{~mm}$ wall thickness, and a bare $\mathrm{W}$ fibre, $1 \mathrm{~m}$ long and $25 \mu \mathrm{m}$ thick. This pendulum configuration is rather insensitive to net forces and to magnetic or gravitational bulk effects, but it maximizes the sensitivity to surface effects, likely the most dangerous for the sensor under developement. The pendulum inertia is $\mathrm{I}=3.48 \mathrm{~g} \mathrm{~cm}^{2}$ and the torsional spring constant is $\Gamma \approx 5.5 \mathrm{nN} \mathrm{m} \mathrm{rad}{ }^{-1}$ with a mechanical quality factor $\mathrm{Q} \approx 3000$. The resulting resonance frequency is $\mathrm{f}_{0} \approx 2 \mathrm{mHz}$.

Any torque $N(\omega)$ acting on the TM can be detected as deflection of the pendulum angular rotation $\phi(\omega)=F(\omega) N(\omega)$, through the transfer function $F(\omega)=$ $\left(\Gamma\left(1-\left(\omega / \omega_{0}\right)^{2}+i / Q\right)\right)^{-1}$. The measured torque noise $S_{N}^{1 / 2}(\omega)=|F(\omega)|^{-1} S_{\phi}^{1 / 2}$, where $S_{\phi}^{1 / 2}$ is the pendulum angular noise, sets upper limits on stray forces $S_{f}^{1 / 2}(\omega)=$ $S_{N}^{1 / 2}(\omega) / R_{\phi}$, making use of a suitable conversion armlength $R_{\phi}$ depending on the specific class of the noise source [6, 7]. Additionally, the high torque sensitivity allows precise measurements of individual noise sources by coherent modulation of the disturbance source itself.

In preparation for the current experimental run, several hardware upgrades were implemented, as shown in figure 1. to improve the pendulum noise performances and allow a detailed investigation of individual force noise sources. The entire pendulum suspension, which is electrically isolated from the TM, has been Aucoated for electrostatic homogeneity. Additionally, tuning screws have been added to minimize the mass quadrupole moment of the rectangular mirror used for the optical readout, reducing possible coupling to gravitational disturbances. Replacement of a stainless steel capillary tube used for holding the pendulum with a $\mathrm{Cu}$ one reduced the 

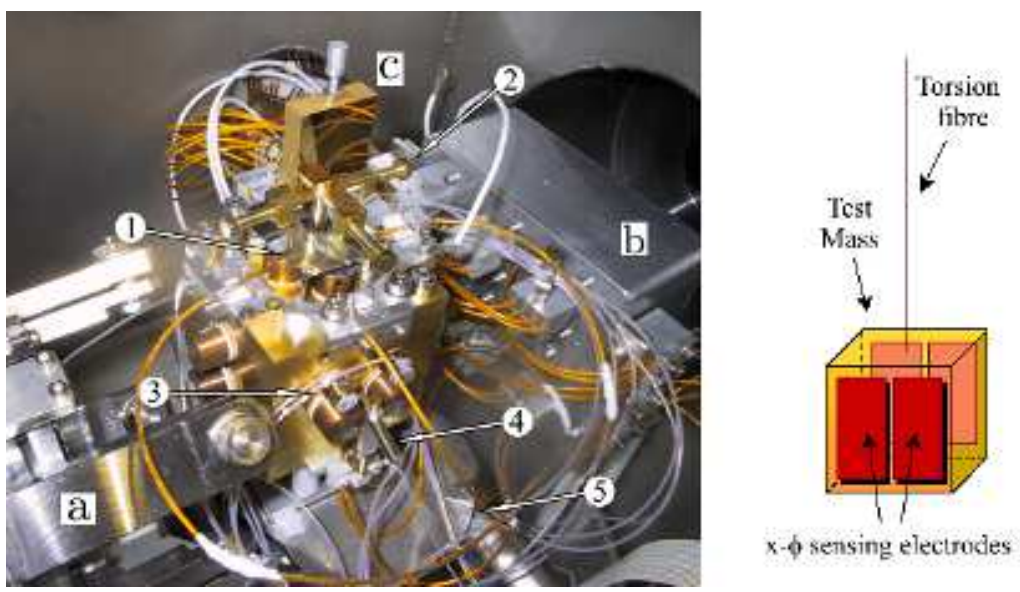

Figure 1. On the left, a photograph of the capacitive sensor inside the vacuum chamber, integrated on the micromanipulator for the positioning (a) and wired with the read-out electronics box (b). The mirror for the independent optical readout is visible (c). Most recent upgrades are labeled: Electrical shields (1); Tunable test mass support (2); Electrical heaters (3); UV light optical fiber (4); Motorized rotary stage (5). On the right, a sketch of the suspended TM surrounded by the $x$ and $\phi$ sensing electrodes.

pendulum's residual magnetic moment to $7 \mathrm{nA} \mathrm{m}^{2}$. Electrical shields cover dielectric surfaces of area $\approx \mathrm{mm}^{2}$ facing the TM support. This shields undesirable stray couplings which have produced a "trans-twist" effect [6, 7], converting sensor-TM relative translation displacement into a torque. The "trans-twist" couplings, $\frac{\partial N}{\partial \theta}$ and $\frac{\partial N}{\partial \eta}$, have been reduced by more than one order of magnitude down to several $\mathrm{nN}$, and thus no longer limit the pendulum noise performance.

The sensor prototype sits on a motorized rotational stage, allowing coherent modulation of the sensor-TM relative rotation angle, needed for the characterization of the overall spring-like couplings. A set of four electrical heaters and five precision thermometers is attached on electrode housing, to induce thermal gradients in order to investigate the related effects. Finally, a simplified discharge system, composed by UV lamps, optical fibers and vacuum feedthroughs has been provided by Imperial College of London, and was integrated in the torsion pendulum facility to test the functionality of the charge control scheme under development for LISA [8].

\section{Characterization of force noise sources}

Upper limits on stray forces. The typical torque sensitivity of the torsion pendulum is plotted in figure 2 For comparison, we also show the instrument limit $S_{N}^{1 / 2}(\omega)=\sqrt{S_{N_{t h}}(\omega)+S_{\phi_{\text {read }}}|F(\omega)|^{-2}}$, where $S_{N_{t h}}^{1 / 2}(\omega)=\sqrt{4 k_{B} T \Gamma /(\omega Q)}$ is the pendulum's intrinsic thermal noise, and $S_{\phi_{\text {read }}}^{1 / 2}|F(\omega)|^{-1}$ is the capacitive sensor angular read-out noise converted into equivalent torque noise. At frequencies above $0.4 \mathrm{mHz}$, measured noise is below $10 \mathrm{fN} \mathrm{m} \mathrm{Hz}{ }^{-1 / 2}$, with a minimum noise floor of $3 \mathrm{fN} \mathrm{m} \mathrm{Hz}{ }^{-1 / 2}$ around $2 \mathrm{mHz}$. The source of the observed excess noise, between a factor 1.5 and 4 from the instrument limit in this frequency range, is currently under 


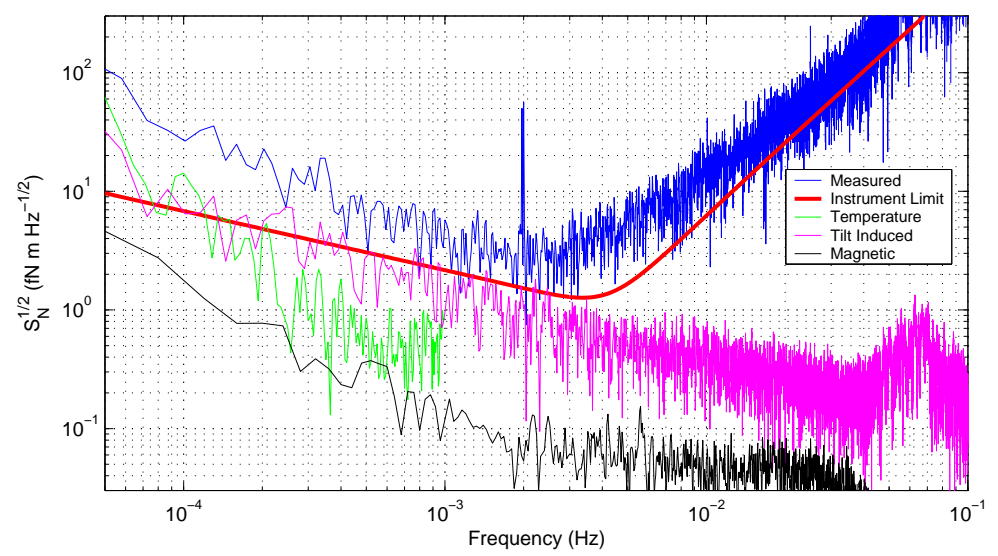

Figure 2. A typical torque noise spectrum of the torsion pendulum (blue line) for a time range of 140000 s. For comparison we show the instrument limit (red), given by pendulum's intrinsic thermal noise in low frequency region, and by the readout noise at higher frequencies. The peak at $2 \mathrm{mHz}$ is artificially produced by the 69000s Hamming window applied to calculate the spectrum. The torque noise induced by temperature fluctuations in the column surrounding the torsion fiber is shown in green. The pink line shows the torque noise induced by the residual "trans-twist" coupling described in [6] 7], while magnetic torque noise is shown in black.

investigation. These data allow us to set significant upper limits on the acceleration noise for LISA. Following [7], we convert the torque noise into acceleration noise as if our hollow $40 \mathrm{~mm} \mathrm{TM}$ were a solid $1.3 \mathrm{~kg} \mathrm{Au} / \mathrm{Pt}$ of the same dimensions. Above $0.4 \mathrm{mHz}$, for a class of noise like circuitry back action, for which $\mathrm{R}_{\phi}=10.25$ $\mathrm{mm}$ (the nominal half separation between adjacent electrodes), acceleration noise is below $1 \mathrm{pm} \mathrm{s}^{-2} \mathrm{~Hz}^{-1 / 2}$, with a minimum about $250 \mathrm{fm} \mathrm{s}^{-2} \mathrm{~Hz}^{-1 / 2}$ around $2 \mathrm{mHz}$. For homogeneously distributed noise sources including random inelastic molecular impacts, where $\mathrm{R}_{\phi}=20 \mathrm{~mm}$, this minimum is $\approx 115 \mathrm{fm} \mathrm{s}^{-2} \mathrm{~Hz}^{-1 / 2}$, about a factor 40 from the LISA goal. At $0.1 \mathrm{mHz}$, the lowest frequency of the LISA band, we estimate a force noise upper limit of order of $1.5 \mathrm{pN} \mathrm{Hz}^{-1 / 2}$ for this class of sources.

Stray DC biases. Stray DC electrostatic fields, due to different work functions of the sensor conducting surfaces, surface contamination or imperfectly shielded external fields, interact with the fluctuating TM charge to produce acceleration noise. Methods to measure and compensate stray DC bias imbalances down to the sub-mV level during the flight missions have been proposed 9] and experimentally verified $\ddagger$ in lab [6]. The measurement technique simulates a sinusoidally varying TM charge by applying dither voltages to selected electrodes and produces on the TM a force (and torque) proportional to the same DC bias imbalances that can produce acceleration noise. The average biases, and thus the resulting force, can be nulled by purposely applying DC compensation voltages to the sensing electrodes through the actuation circuitry. This allows a reduction of DC biases of a factor $\approx 100$ compared to measured uncompensated values, that we found to be typically of order tens of $\mathrm{mV}$ across the sensor housing, and yields a suppression of the related acceleration noise down to a

$\ddagger$ For LISA, the relevant DC bias imbalance is the average translational potential difference across the sensor $\Delta_{x}$, whereas with the pendulum we measure the analogous rotational imbalance $\Delta_{\phi}[9$. 


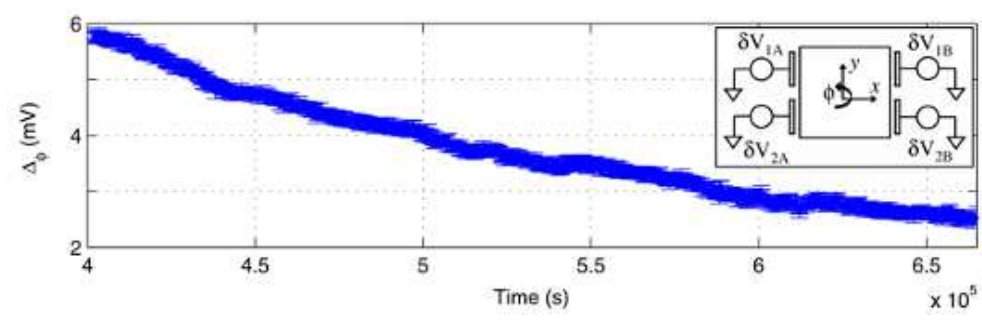

Figure 3. Time series of a 3 days measurement of one of the stray DC bias combinations, namely $\Delta_{\phi}=\left(\delta V_{1 a}+\delta V_{2 b}-\delta V_{2 a}-\delta V_{1 b}\right)$ as shown in the inset and defined in 9. A compensation of the $-62.8 \mathrm{mV}$ average imbalance is applied.

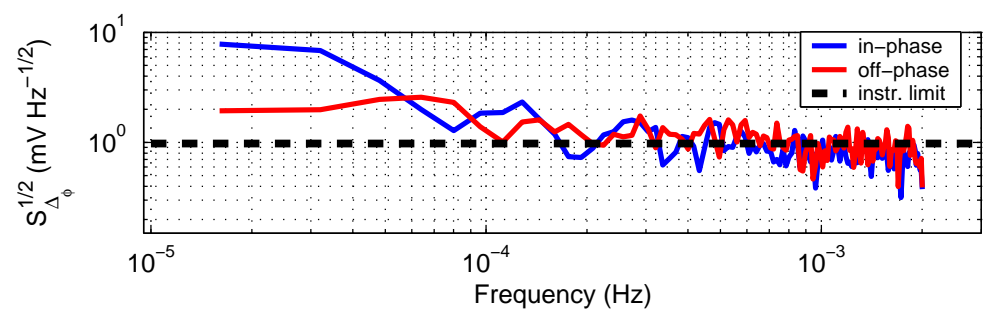

Figure 4. Noise spectrum of the DC bias time series showed in figure 31000 data points, corresponding to 250000s, are analyzed using a 62500s Hamming window. As described in the text, the in-phase demodulated signal (blue line), which carries the $\Delta_{\phi}$ information, and off-phase (red line) signal, which should contain only noise, show up with the same noise levels down to $0.1 \mathrm{mHz}$. The instrument limit is shown for comparison (black dashed line), and is given by the pendulum torque noise at the measurement frequency of $4 \mathrm{mHz}$.

negligible level for LISA.

During this experimental campaign, we performed a complete investigation of all DC bias imbalances characteristic of the Mo-Shapal sensor prototype under testing. In addition to the net DC bias imbalances, performance limiting effects can also arise in the DC bias fluctuations. As described in [11 for the case of fluctuating actuation voltages, a fluctuation in the stray voltage imbalance $\Delta_{x}$, along the LISA sensitive axis, mixes with the TM net charge $q$ to produce force noise on the TM $S_{F}^{1 / 2} \approx \frac{\partial C}{\partial x} \frac{q}{C_{T}} S_{\Delta_{x}}^{1 / 2}$, with $\frac{\partial C}{\partial x}$ and $C_{T}$ defined in [9]. Additionally, unstable elements of $\Delta_{x}$ mix with the individual biases themselves to produce force noise. The LISA requirement for fluctuating voltages $\approx 10 \mu \mathrm{V} \mathrm{Hz}^{-1 / 2}$, which produces a maximum acceleration noise $\approx 0.2 \mathrm{fm} \mathrm{s}^{-2} \mathrm{~Hz}^{-1 / 2}$ with both charge and net DC bias contributions taken into account 11], can not be measured yet at this level with this pendulum. An example of measurement of the long term stability of the DC bias imbalance $\Delta_{\phi}$, which is the rotational analogous of $\Delta_{x}$, is plotted in figure 3. The related noise spectrum is shown in figure 4 where the in-phase demodulated signal, which carries the $\Delta_{\phi}$ information, and the off-phase one, which should contain only noise, are analyzed. An upper limit can be set at $1 \mathrm{mV} \mathrm{Hz}^{-1 / 2}$, a factor 100 over the LISA requirement. For frequencies higher than $0.1 \mathrm{mHz}$, the measured noise is limited by the pendulum torque noise at the measurement frequency of $4 \mathrm{mHz}$. The excess voltage noise at lower frequency is indicative of an observed non-stationary drifting of the DC bias, as illustrated in 
figure 3 This excess noise does not depend on the applied compensation voltages, nor is related with possible fluctuations of the dithering voltages used to perform the measurement. To investigate a possible correlation, DC bias measuremenst have been performed at different temperatures and appling different temperature gradients. No clear sistematic dependence have been observed in both cases, but changing temperature seems to destabilize the net imbalance $\Delta_{\phi}$. Currently, an aggressive investigation is ongoing to better understand the origin of these stray DC voltages and their fluctuations, as well as their spatial distribution in the sensor.

Test Mass Charge Control. In LISA, the TMs become electrically charged by cosmic ray particle radiation, undergoing Coulomb forces for the interaction with surrounding and nominally grounded surfaces and Lorentz forces due to its motion in the interplanetary magnetic field. The method to actively control the charge uses the emission of photoelectrons by UV light in combination with suitable electron driving voltages [8]. A charge measurement technique, where dither voltages are applied to the sensing electrodes to produce a coherent torque proportional to the TM voltage drop $V_{M}$ to the grounded sensor housing 9], has been successfully tested with the torsion pendulum facility. Using a typical modulation frequency $\mathrm{f}_{m}=5 \mathrm{mHz}$, and a dither bias amplitude of $50 \mathrm{mV}$, a 3 hour measurement allows to resolve $V_{M}$ at the sub-mV level, corresponding to $\approx 5000$ elementary charges. This resolution is well below the level of $10^{6}$ elementary charges envisioned as a discharge threshold, before the charge-induced stiffness, to be discussed in the next section, becomes dominant [1]. Furthermore, a preliminary test of this discharging scheme has been performed using the UV light hardware provided by the Imperial College of London. The UV light fiber is aligned to illuminate both the suspended TM and one of the sensing electrodes. The two photoelectron currents emitted from both surfaces can be driven from the TM to the electrode or viceversa by purposely applying bias voltages to the electrode plates. Preliminary tests, performed with a crude fibre alignment and with a simple on/off control of the UV light intensity, demonstrated the capability of bipolar charge transport and control. The observed charge transfer rate is at least of order of $10^{5}$ elementary charges per second. The lower limit is imposed by the slow sampling time $(\approx 200 \mathrm{~s})$ of the charge measurement and by the slow torsion pendulum dynamics.

Stiffness. As shown in (11), spacecraft-TM springlike couplings ("stiffness") $k_{p}$ converts any residual relative spacecraft motion into force noise on the TM. As discussed in [2, 3], electrostatics play a leading role among the possible stiffness sources, and the likely dominant contribution is given by the $100 \mathrm{kHz}$ sensing bias of the capacitive readout. The rotational analogous of the sensing stiffness has been already measured and found to agree with theoretical predictions at the $10 \%$ level [6. 10, nevertheless it is also important to search for any unmodeled coupling arising in the sensor.

An example of the measurement technique developed to investigate the total rotational stiffness $\Gamma_{s}$ induced by the GRS is given in figure 5] Square wave modulation of the sensor angle $\phi_{\text {sens }}$, by means of the rotary stage, induces a torque $N(t)=-\Gamma_{s}\left(\phi-\phi_{\text {sens }}(t)\right)$ on the pendulum. The torque amplitude, and thus the stiffness itself, is measured by the coherent deflections of the pendulum angular rotation.

As shown in figure [5 the total stiffness has been measured both with the sensing bias on and off. By the difference of the two measurements, the sensing bias stiffness contribution results to be $(-89.2 \pm 0.4) \mathrm{pN} \mathrm{m} \mathrm{rad}{ }^{-1}$ in agreement with previous results [6]. Besides, a residual stiffness $\Gamma_{\text {res }}=(-11.6 \pm 0.2) \mathrm{pN} \mathrm{m} \mathrm{rad}^{-1}$ has been measured. 

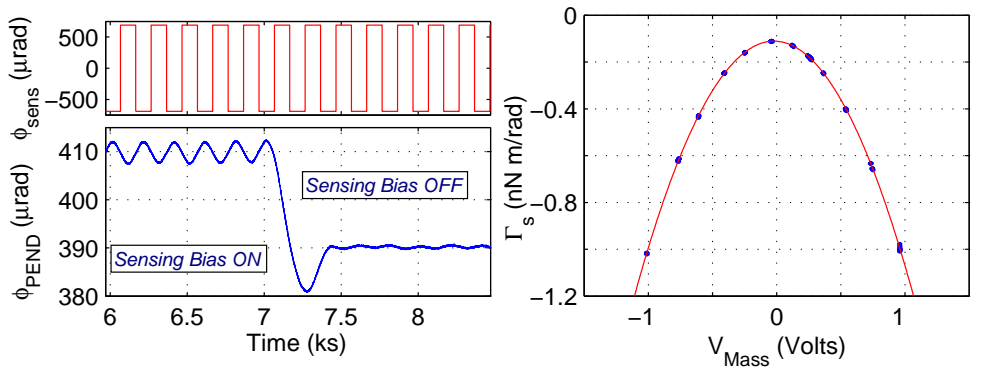

Figure 5. Left Panels: A sequence of two measurements of the total rotational stiffness, respectively with the sensing bias on and off. On top, the sensor rotation angle about the suspended test mass, as read by the motorized stage encoder, modulated at $5 \mathrm{mHz}$. On the bottom, the pendulum motion, where the free pendulum oscillation and higher harmonics of the modulation frequency are filtered. The pendulum coherent response, as well as the equilibrium position, change when removing the sensing bias stiffness. Right panel: Measurement of the rotational stiffness as function of the TM voltage drop to the sensor housing (blue dots). The red line shows the quadratic fit. Uncertainties for each stiffness data point are about $\approx 1 \mathrm{pN} \mathrm{m} \mathrm{rad}-1$.

This unpredicted coupling could be explained by patch voltages of order of $100 \mathrm{mV}_{r m s}$ homogenously distributed across the sensor housing. Note that this rms value is consistent with the average DC bias typically measured. Another possible explanation is a residual of the "trans-twist" interaction, through a coherent sensor translational displacement of order of $\mu \mathrm{m}$ associated with mrad angular motion, due to mounting misalignment. A further analysis is ongoing to investigate the source of this residual stiffness.

A potentially relevant contribution to the stiffness arises from Coulomb forces between the electrically charged TM and the surrounding sensor housing conduction surfaces. As for the sensing bias, the measurement of the charge dependent stiffness is a key verification of the sensor electrostatic model. Measurements described in the previous paragraph were performed holding $V_{M}=0 \mathrm{~V}$. For this investigation, the TM has been biased to different voltage drops $V_{M}=q / C_{T}$ exploiting its finite discharge time $(\approx 10$ hours $)$ through the rest of the pendulum, by directly biasing the $\mathrm{W}$ torsion fiber $^{+}$. Results of this investigation are shown in figure 5 The measured coupling $\Gamma_{s}\left(V_{M}\right)=-\frac{\partial N}{\partial \phi}=-\frac{1}{2} \sum_{i}\left(\frac{\partial^{2} C_{i}}{\partial \phi^{2}}\right) V_{M}^{2}$, where $\sum_{i}\left(\frac{\partial^{2} C_{i}}{\partial \phi^{2}}\right)=(1.84 \pm 0.01) \mathrm{nF} \mathrm{rad}^{-2}$ is the sum of the second derivatives of all electrode capacitances $C_{i}$ with respect to the $\mathrm{TM}$, is in agreement within $10 \%$ with the F.E.M. prediction [10. It is worth noting that this estimate is not completely independent from the model prediction, because of the assumption of $\frac{\partial C_{x}}{\partial \phi} \simeq 11.5 \mathrm{pF} \mathrm{rad}{ }^{-1}$, estimated with F.E.M analysis, which enters in the calibration of the charge measurement.

The maximum stiffness* is found for $V_{M}=(-20.2 \pm 0.3) \mathrm{mV}$, rather than $V_{M}=0$ $\mathrm{V}$ as one might expect. This is likely due to the difference between the "zero" defined by average potential of the four electrodes relevant to the charge measurement and

+ This finite discharge time would require continuous charge control in order to hold $V_{M}$ fixed. To avoid the electrostatic stiffness associated with electron driving voltage affecting the measurements, the UV light charge control scheme is not employed in this experiment.

* As $\Gamma_{s}$ is negative, the maximum value corresponds to the minimum coupling. 


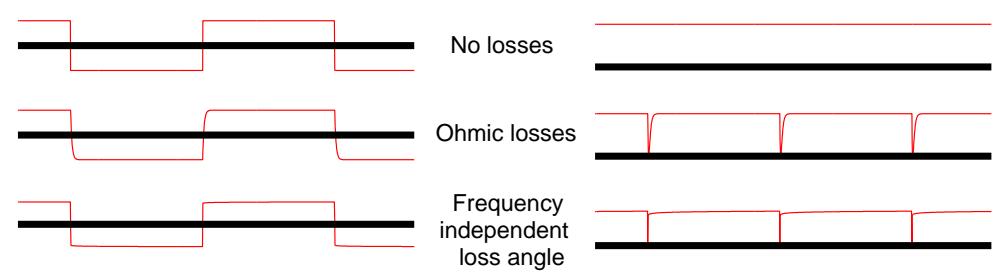

Figure 6. Correspondence between voltage applied $V(t)$ (left) and torques $N(t) \propto V^{2}(t)$ induced on the pendulum (right) in the case of zero loss, ohmic loss and frequency independent loss angle.

the weighted average of the potential on all conducting surfaces for which $\frac{\partial^{2} C_{i}}{\partial \phi^{2}} \neq 0$ (relevant to the stiffness measurement). This $20 \mathrm{mV}$ difference is consistent with measured levels of DC biases in the sensor.

Beyond the successfull confirmation of the reliability of the electrostatic model, it is worth nothing that $\mathrm{a} \approx 100 \mathrm{mV}_{\text {rms }}$ stray $\mathrm{DC}$ voltage, a possible cause of the measured residual stiffness, would produce a translational stiffness in LISA of order of $\approx 10 \mathrm{nN} \mathrm{m}^{-1}$. This is about 10 times smaller than the dominant contributions envisioned, and thus it would not create a significant noise source for the scientific mission.

Dielectric Losses. Voltage thermal noise originates in dielectric lossy layers on electrodes and TM surfaces, characterized by a dielectric loss angle $\delta$, and couples to DC voltages to produce force noise on the TM itself. In order to keep this contribution whithin $10 \%$ of the LISA acceleration noise budget, the maximum allowed is $\delta \approx 10^{-5}[11$.

Up to now, our attempts to measure $\delta$ through its effect on the pendulum $\mathrm{Q}$, as in 12, have been unreproducibile at a level which prevents the extraction $\delta$ to better than $10^{-4}$. To overcome this limit, we developed a new measurement technique, which takes advantage of the transient produced on square wave voltages applied on a lossy conductor surface. The principle of this technique is schematized in figure 6 In the lossless ideal case, a square wave voltage, applied to the sensing electrodes, would induce a constant torque $N(t) \propto V^{2}(t)$ on the TM. In the presence of losses, torque transients are produced coherently with each voltage transition. The torque results in a train of pulses, whose amplitude is strictly related to the loss itself, at twice the voltage carrier frequency.

A first calibration of the method has been performed by adding a resistor to create a true ohmic loss, characterized by a frequency independent time delay $\tau$. For the case of a frequency independent loss angle (for which $\tau \propto \omega^{-1}$ ), a synthetized time series simulating the waveform for a fixed $\delta$ has been used to calibrate. In both cases, resulting measured torques are in agreement with expected values with accuracy below the $10 \%$ level. With the sensitivity shown in figure 2 the method has a resolution of the order of $10^{-16} \mathrm{~N} \mathrm{~m}$ for a few hour measurement and permits extraction of $\delta$ to within $10^{-6}$.

Measurements have been performed on different electrode pairs, for voltage amplitudes from $1 \mathrm{~V}$ to $3 \mathrm{~V}$, and at different frequencies between 0.2 and $3 \mathrm{mHz}$. Preliminary results give an estimate of $\delta \approx 10^{-6}$, over the frequency range of investigation. A more detailed model of ohmic and dielectric losses in the sensor is needed in analyzing theese measurements. Still, it is worth noting that these values 

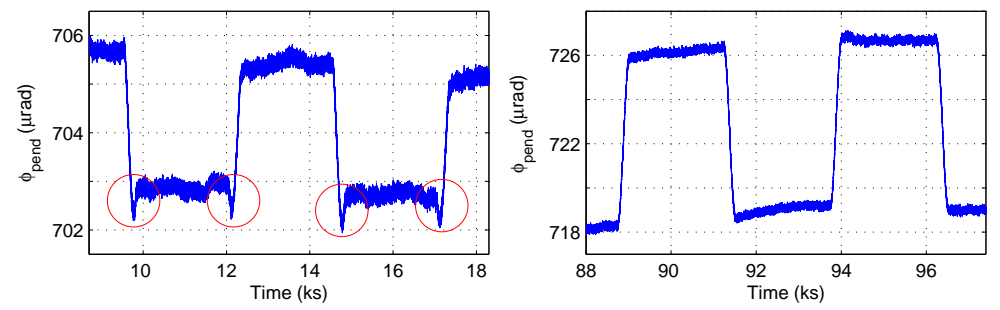

Figure 7. Examples of the dielectric loss angle measurement technique. In both cases, the free pendulum mode signal is filtered and the $0.2 \mathrm{mHz}$ signal is due to uncompensated DC bias. The left panel shows a calibration performed using ohmic losses (corresponding to $\tau \approx 10 \mathrm{~ms}$ ): the pendulum response at the torque transient produced at $0.4 \mathrm{mHz}$ is highlighted with red circles. The right panel shows the pendulum response during a sensor related $\delta$ measurement: the small signal, measured by coherent demodulation at $0.4 \mathrm{mHz}$ and corresponding to $\delta \approx 10^{-6}$, is here not readily visible.

are one order of magnitude less than the maximum dielectric losses allowed for LISA. Thermal Gradient Related Effects. Temperature gradients $\Delta T$ across the sensor housing, with spectral density $S_{\Delta T}^{1 / 2}$, are expected to cause noisy forces onto the TM surface area $\mathrm{A}$, according to the following formula:

$$
S_{F_{\Delta T}}^{1 / 2}=\left(\frac{8 \sigma}{3 c} A T^{3}+\frac{A}{4} \frac{P}{T}+\frac{A}{4} G \mathcal{Q} \frac{\Theta}{T^{2}}\right) S_{\Delta T}^{1 / 2}
$$

Here, the first two terms are related, respectively, to the radiation pressure effect, where $\sigma$ is the Stefan-Boltzmann constant and $c$ is the speed of light, and to the radiometric effect, linearly proportional to the average pressure $P$ inside the sensor, which can be greater than the pressure in the vacuum chamber due to the outgassing of the electrode housing internal surfaces. The last term is due to temperature dependent outgassing, causing pressure difference across the TM proportional to the temperature gradient $\Delta T$. Here $\mathcal{Q}$ is the outgassing rate inside the sensor, scaled by the geometrical factor $G$ resulting from a combination of the conductance of the paths around the test mass and through the holes in the electrode housing walls, and $\Theta$ is an effective activation temperature of the outgassing phenomena. With a temperature gradient stability of $S_{\triangle T}^{1 / 2} \approx 10 \mu \mathrm{K} \mathrm{Hz}^{-1 / 2}$, the current LISA error budget tentatively apportions an acceleration noise $\approx 0.5 \mathrm{fm} \mathrm{s}^{-2} \mathrm{~Hz}^{-1 / 2}$ [11, almost equally distributed among the different envisioned contributions. The uncertainty on this estimate arises mainly on the lack of knowledge of the parameters $\Theta$ and $\mathcal{Q}$, which necessitates an experimental investigation, which would, moreover, highlight any other possible unexpected thermal related disturbance.

The contributions from each effect should be distinguishable by its specific dependence on pressure and temperature. In particular, any pressure independent signal larger that the radiation pressure effect would represent an important upper limit for the less known outgassing related effects. A preliminary investigation has been performed here by applying off-axis "rotational" temperature differences that convert the forces described above into torques measurable with this pendulum (see figure 8) The results have been compared with the prediction of a simplified model that allows for converting the forces described in (2) into torques. This model assumes that the effects are isotropic (dependent just on the local temperature) and a rough estimation 

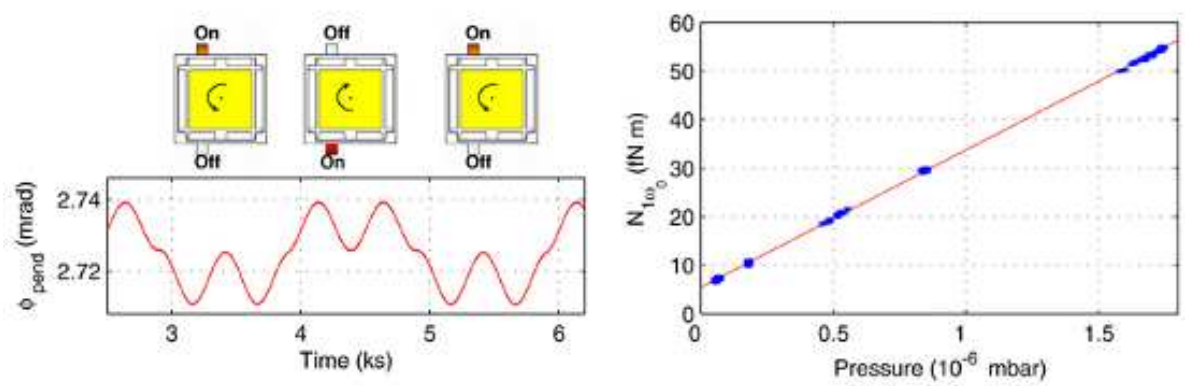

Figure 8. Left panels: an example of the pendulum coherent response (bottom) to the heat of 0.2 Watt alternative applied to opposing electrodes every $1000 \mathrm{~s}$ (top). We roughly estimated that the consequent temperature distribution inside the sensor is equivalent, from the point of view of the torque produced, to the presence of $70 \mathrm{mK}$ temperature drop between the corresponding two opposite electrodes (with an uncertainty of a factor 2). Right Panel: Demodulated torques at the carrier frequency of $0.5 \mathrm{mHz}$ measured as function of the residual pressure in the vacuum vessel. The average sensor temperature for these measurements is $\mathrm{T} \approx 295 \mathrm{~K}$.

of the temperature distribution inside the sensor, based on the measurement performed by a thermometers array positioned on its external surfaces (its accuracy will improve thanks to the currently in progress thermal F.E.M. of the sensor). Measurements of torque, coherently induced by alternatively applying $0.2 \mathrm{~W}$ to the heaters behind two opposing electrodes at a frequency of $0.5 \mathrm{mHz}$, are shown in figure 8 as function of the pressure measured inside the vacuum vessel. The linear dependence is consistent with the presence of the radiometric effect, which is the leading term above $\approx 2 \times 10^{-7}$ mbar. The observed slope is $2.8 \times 10^{-8} \mathrm{~N} \mathrm{~m} \mathrm{mbar}{ }^{-1}$ and is compatible with our rough prediction of $3 \times 10^{-8} \mathrm{~N} \mathrm{~m} \mathrm{mbar}{ }^{-1}$ for radiometric effect, affected by an inaccuracy of a factor 2. The pressure independent terms show up in the positive intercept at zero pressure: being the expected radiation pressure responsible for half of it, the unknown outgassing related effects turn out to give a comparable contribution. These results are in line with of the assumption of the current LISA noise budget, and show that there are no contributions, from outgassing or other effects, that are likely to be present at levels well in excess of the radiation pressure and radiometric effects. However, it is advisable to confirm them with a more detailed and accurate investigation with a four masses pendulum configuration [13], which is sensitive directly to the net forces acting on the TM. Such a measurement is less model dependent and guaranties more accurate estimation of a possibly non-uniform outgassing contribution.

\section{Conclusions}

In the near future we will use the torsion pendulum facility to characterize a new implementation of GRS, which is presently in preparation, employing the current LTP geometry design for position sensor and TM (4mm TM-sensing electrodes gaps and 46mm TM cubic size, different electrode configuration [2]).

Additionally, a four mass pendulum facility [13, which is currently under development and that will be built in few months, will allow a more representative characterization of the GRS related force disturbances along the translational axis of 
relevance for LISA.

\section{Acknowledgments}

The authors would like to thank T.J.Sumner, G.K.Rochester and D.N.A. Shaul at the Imperial College of London for their collaboration in suppling the charge management hardware and electrostatic modelling of the sensor. This work was supported by ESA, INFN and ASI.

\section{References}

[1] Bender P et al, LISA ESA-SCI(2000)11, 2000

[2] Weber W J et al 2002 SPIE Proc. 485631

[3] Dolesi R et al 2003 Class. Quantum Grav. 20 S99

[4] Bortoluzzi et al 2004 Class. Quantum Grav. 21 S573

[5] Hueller M et al 2002 Class. Quantum Grav. 191757

[6] Carbone L et al 2003 Phys. Rev. Lett. 91151101

[7] Carbone L et al 2004 Class. Quantum Grav. 21 S611

[8] Sumner T J et al 2004 Class. Quantum Grav. 21 S597

[9] W J Weber et al 2003 Advances in space research Preprint gr-qc/0309067 (at press)

[10] Shaul D N A et al 2002 SPIE Proc. 485643

[11] Stebbins R T et al 2004 Class. Quantum Grav. 21 S653

[12] Speake C C et al 1999 Phys. Lett. A 263219

[13] Carbone L et al Proc. of $10^{\text {th }}$ Marcel Grossmann Meeting on General Relativity Preprint gr-qc/0411049 (at press) 